\title{
Avaliação cardiovascular perioperatória em cirurgias não cardíacas como ferramenta para redução de risco de eventos cardíacos adversos
}

\section{Perioperative cardiovascular evaluation for noncardiac surgery as a tool to reduce risk of adverse cardiac events}

Daniel Rocha e Silva ${ }^{1}$. Fernanda Paula Cavalcante ${ }^{2}$. Flávio Lobo Maia ${ }^{3}$. Lorena Antônia Sales de Vasconcelos Oliveira $^{3}$. Cláudia Regina Fernandes ${ }^{4}$.

1 Residente de Anestesiologia do Hospital Universitário Walter Cantídio, Universidade Federal do Ceará (UFC), Fortaleza, Ceará, Brasil. 2 Anestesiologista, chefe do Centro de Ensino e Treinamento em Anestesiologia do Hospital Universitário Walter CantídioUFC, Fortaleza, Ceará, Brasil. 3 Anestesiologista, corresponsável pelo Centro de Ensino e Treinamento em Anestesiologia do Hospital Universitário Walter Cantídio-UFC, Fortaleza, Ceará, Brasil. 4 Anestesiologista, professora da Faculdade de Medicina, Universidade Federal do Ceará (UFC), chefe da Divisão de Apoio Diagnóstico e Terapêutico do Hospital Universitário Walter Cantídio-UFC, Fortaleza, Ceará, Brasil.

\section{RESUMO}

Complicações cardíacas estão entre as principais causas de morbimortalidade no perioperatório. Adequada avaliação cardiovascular, portanto, é fundamental para a identificação de patologias e potenciais fatores de risco e, quando necessário, otimização clínica do paciente no perioperatório. Sendo assim, tem-se, com esse estudo, o objetivo de revisar as mais recentes recomendações de avaliação cardiovascular e de estratégias para redução na incidência de eventos cardíacos adversos no perioperatório de cirurgias não cardíacas. Para esse fim, realizou-se revisão de literatura na base de dados MEDLINE (PubMed). Tornando mais aplicáveis os instrumentos de avaliação cardiovascular, contribui-se para redução na incidência de morbimortalidade perioperatória associada a eventos cardíacos adversos, redução de custos com hospitalização e solicitação abusiva de exames complementares, além de menores taxas de suspensão de procedimentos eletivos.

Palavras-chave: Cuidado perioperatório. Doenças cardiovasculares. Infarto do miocárdio.

\section{ABSTRACT}

Cardiac complications are among the leading causes of morbidity and mortality in the perioperative period. Adequate cardiovascular evaluation, therefore, is critical to the identification of cardiac diseases and potential risk factors and, when appropriate, clinical optimization of the patient in the perioperative period. This study aims to revise the latest recommendations for cardiovascular assessment and strategies to reduce the incidence of adverse cardiac events in the perioperative period of non-cardiac surgery. To this end, it carried out a literature review in MEDLINE (PubMed). Making it applicable to cardiovascular assessment tools, it may contribute to reduction in the incidence of morbidity and mortality associated with adverse cardiac events, to reduce hospitalization costs and excessive complementary exams, and to also a reduction in elective procedures suspension rates.

Keywords: Perioperative care. Cardiovascular diseases. Myocardial infarction.

Autor correspondente: Daniel Rocha e Silva, Rua Mariana Furtado Leite, 1250, apartamento 1002, Engenheiro Luciano Cavalcante, Fortaleza, Ceará. CEP: 60811-150. Telefone: +55 85 99600-3535. E-mail: danielrs07@gmail.com

Conflito de interesses: Não há qualquer conflito de interesses por parte de qualquer um dos autores.

Recebido em: 14 Nov 2015; Revisado em: 08 Mar 2015; Aceito em: 22 Mar 2015. 


\section{INTRODUÇÃO}

Complicações cardiovasculares estão entre as principais causas de morbimortalidade no perioperatório. Dentre os pacientes que serão submetidos a cirurgias não cardíacas, a minoria apresenta risco cardíaco elevado, sendo importante seu correto diagnóstico.

Uma adequada avaliação cardiovascular é fundamental para a identificação de potenciais fatores de risco e de patologias e, quando necessário, otimização clínica do paciente no perioperatório. Essa avaliação, portanto, deve ser eficaz e, ao mesmo tempo, prática. Deve considerar contexto cirúrgico (cirurgia de emergência, urgência e tempo sensível), exame clínico do paciente, estratificação de risco cardíaco e necessidade de exames diagnósticos complementares.

\section{MATERIAL E MÉTODOS}

Trata-se de revisão de literatura, realizada a partir de seleção de artigos nacionais e internacionais através do banco de dados MEDLINE (PUBMED). Foram selecionados artigos publicados nos últimos 18 anos com enfoque na avaliação cardiovascular perioperatória em cirurgias não cardíacas. Como descritores foram utilizados os seguintes termos, escritos na língua inglesa: perioperative care, cardiovascular diseases, myocardial infarction, noncardiac surgery e major adverse cardiac event.

As recomendações desta revisão foram extraídas do Guideline on perioperative cardiovascular evaluation and management of patients undergoing noncardiac surgery, publicadas em 2014 pelo American College of Cardiology (ACC) Foundation/American Heart Association (AHA). Foram selecionadas as recomendações com os mais altos níveis de evidência (Quadro 1).

A pesquisa incluiu artigos originais, artigos de revisão e diretrizes escritos na língua inglesa e portuguesa. Foram utilizados 21 artigos, sendo 1 nacional e 20 internacionais.

Quadro 1. Níveis de evidência e classes de recomendação. ${ }^{2}$

\begin{tabular}{|l|l|l|l|l|}
\hline Níveis de Evidência & \multicolumn{2}{l|}{ Classes de Recomendação } \\
\hline Nível A & $\begin{array}{l}\text { Resultados derivados de múltiplos } \\
\text { ensaios clínicos randomizados ou de } \\
\text { metanálises ou de revisões sistemáticas }\end{array}$ & Classe I & Benefício >>>risco & $\begin{array}{l}\text { Procedimento/tratamento ou } \\
\text { teste terapêutico é recomendado }\end{array}$ \\
\hline Nível B & $\begin{array}{l}\text { Resultados derivados de um único } \\
\text { ensaio clínico randomizado ou de } \\
\text { estudos controlados não randomizados }\end{array}$ & Classe IIa & Benefício >>risco & $\begin{array}{l}\text { Pode ser aplicado devido à sua } \\
\text { performance }\end{array}$ \\
\hline Nível C & $\begin{array}{l}\text { Resultados baseados em séries de casos } \\
\text { ou diretrizes baseadas na opinião de } \\
\text { especialistas }\end{array}$ & Classe IIb & Benefício $\geq$ risco & Pode ser considerado \\
\hline Nível D & $\begin{array}{l}\text { Coorte histórica, não randomizada ou } \\
\text { estudo de caso controle }\end{array}$ & Classe III & Risco $\geq$ benefício & Pode ser prejudicial \\
\hline
\end{tabular}

Fonte: elaborado pelos autores.

\section{Fatores de risco clínicos}

\section{Doença arterial coronariana}

A incidência de morbidade pós-operatória relativa à doença arterial coronariana (DAC) depende da definição considerada, que varia desde a elevação isolada dos níveis séricos de marcadores de lesão miocárdica à definição mais clássica, que envolve outros sinais de isquemia. ${ }^{2}$

Infarto miocárdico subclínico é comum no perioperatório. Mais de 50\% dos casos podem permanecer sem diagnóstico se os médicos atentarem apenas aos sinais e sintomas clássicos. ${ }^{3}$

Grande parcela dos eventos isquêmicos no perioperatório ocorrem dentro dos 3 primeiros dias após a cirurgia, ${ }^{4}$ período no qual a maioria dos pacientes recebem analgesia. Além disso, uma pequena fração dos pacientes, porém de alto risco, permanecerá intubada e sedada durante este período, o que limita a comunicação dos sintomas. Vale ressaltar que muitos dos sinais e sintomas experimentados na vigência do infarto agudo do miocárdio (IAM) têm etiologia comum com eventos frequentes no pós operatório (atelectasia, pneumonia, hipovolemia, sangramento, efeitos colaterais de medicações), retardando ainda mais o diagnóstico precoce.

Situação comum na prática diária é a realização de cirurgias em pacientes com histórico de infarto recente (ocorrido nos últimos 6 meses). Estudo retrospectivo de revisão de prontuários concluiu que, na ausência de intervenção coronariana, somente após 60 dias do evento isquêmico, 
o paciente poderá ser submetido a procedimento cirúrgico eletivo não cardíaco com segurança. ${ }^{5}$

\section{Insuficiência cardíaca}

Pacientes com insuficiência cardíaca (IC) descompensada ou simples histórico de IC estão sob significativo risco de complicações perioperatórias, sobretudo falência miocárdica. ${ }^{6,7}$ Pouco valorizada por muitos profissionais, a disfunção diastólica, isolada ou combinada à disfunção sistólica do ventrículo esquerdo (VE), tem sido associada a aumento na taxa de eventos cardíacos adversos (ECA), no tempo de internação e na mortalidade. ${ }^{8}$

Fração de ejeção do ventrículo esquerdo (FEVE) inferior a $30 \%$ é fator de risco isolado para morbimortalidade no pósoperatório em pacientes portadores de IC. ${ }^{9}$

Embora os modelos de estratificação de risco cardiovascular no perioperatório destaquem $\mathrm{DAC}$ em relação à $\mathrm{IC}$, pacientes com IC descompensada estão sob maior risco de morte no pósoperatório. Daí a importância da estabilização dos pacientes com essa patologia. ${ }^{2}$

\section{Cardiomiopatias}

3.1 Cardiomiopatia restritiva: o débito cardíaco (DC) depende da pré-carga e da frequência cardíaca. Assim, redução da volemia ou das pressões de enchimento, bradicardia ou taquicardia e arritmias atriais não são bem toleradas. ${ }^{2}$

3.2 Cardiomiopatia hipertrófica obstrutiva: queda na resistência vascular sistêmica, perdas sanguíneas, redução da pré-carga ou das pressões de enchimento do VE podem reduzir o enchimento diastólico e o DC, com potenciais resultados indesejados. $^{2}$

3.3 Cardiomiopatia periparto: rara causa de cardiomiopatia dilatada, ocorre em aproximadamente $0,1 \%$ das gestações, manifestando-se durante os últimos meses da gravidez e nos 6 primeiros meses de pós parto. Nestes pacientes, devese otimizar a administração de fluidos e evitar depressão miocárdica. Na maioria dos casos, as pacientes mantém-se estáveis e a recuperação é plena. ${ }^{2}$

\section{Valvopatias}

Portadores de valvopatias são frequentes no contexto das avaliações pré-operatórias, principalmente devido o aumento da expectativa de vida da população geral. Sendo assim, identificação através de exames clínico e ecocardiográfico é de grande valor para a redução dos riscos de ECA no perioperatório.

Recomenda-se realização de ecocardiograma pré-operatório em pacientes com suspeição clínica de estenose ou regurgitação valvar, moderada a grave: a) na ausência de ecocardiograma nos últimos 12 meses ou b) se houver alteração clínica significativa em relação à última avaliação ${ }^{10}$ (Classe I - Nível de evidência: C).

Para pacientes com indicação de abordagem cirúrgica valvar e, ao mesmo tempo, candidatos à cirurgia eletiva não cardíaca, a realização inicial da intervenção valvar é eficaz na redução do risco perioperatório (Classe I - Nível de evidência: C).

A incidência de IAM foi mais frequente em pacientes com estenose aórtica (EA) que na população geral submetida a cirurgias eletivas não cardíacas $(3,0 \%$ versus $1,1 \%$; $\mathrm{P}=0.001) .{ }^{11}$

Recomenda-se monitorização hemodinâmica no intraoperatório e no pós operatório de cirurgias eletivas não cardíacas de alto risco em pacientes portadores de EA severa, mesmo se assintomáticos (Classe IIa - Nível de evidência: B), bem como de pacientes portadores de estenose mitral (Classe IIb - Nível de evidência: C) e regurgitação valvar (Classe IIa Nível de evidência: $\mathrm{C}$ ).

\section{Arritmias e distúrbios de condução}

A presença de arritmias no perioperatório deve ser prontamente investigada em relação à doença cardiopulmonar subjacente, isquemia miocárdica, toxicidade exógena ou distúrbios metabólicos, a depender da história clínica, natureza e tempo de instalação da arritmia. ${ }^{2}$

\section{Doença vascular pulmonar}

Terapia vascular pulmonar crônica em pacientes com hipertensão pulmonar que serão submetidos à cirurgia não cardíaca deve ser mantida, exceto se contraindicada ou não tolerada (Classe I - Nível de evidência: C).

\section{Definindo o risco cardíaco}

Instrumentos validados de previsão de risco podem ser úteis em prever a ocorrência no perioperatório de ECA em pacientes submetidos à cirurgias não cardíacas (Classe IIa - Nível de evidência: B).

Existem inúmeras classificações de risco de ECA, porém, atualmente, a mais utilizada é o índice de risco cardíaco revisado (IRCR), ou escore de Lee revisado (Quadro 2). Validado e de simples aplicação, é amplamente aceito para avaliação de risco de complicações. ${ }^{12}$ Contém seis preditores de risco para eventos cardíacos adversos no perioperatório, somente um dos quais é baseado no procedimento cirúrgico. Pacientes com até 1 preditor são classificados como baixo

Quadro 2. Índice de risco cardíaco revisado. ${ }^{12}$

\begin{tabular}{|l|}
\hline \multicolumn{1}{|c|}{ Critérios } \\
\hline Creatinina $\geq 2 \mathrm{mg} / \mathrm{dL}$ \\
\hline Insuficiência cardíaca \\
\hline Diabetes mellitus insulino dependente \\
\hline Cirurgia vascular suprainguinal, intra-abdominal, intratorácica \\
\hline $\begin{array}{l}\text { História de acidente vascular cerebral ou ataque isquêmico } \\
\text { transitório }\end{array}$ \\
\hline Doença cardíaca isquêmica \\
\hline
\end{tabular}

Fonte: elaborado pelos autores. 
risco para ECA $(<1 \%)$. Pacientes com 2 ou mais preditores são classificados como alto risco $(>1 \%)$.

\section{Capacidade funcional}

A capacidade funcional é um razoável preditor de ECA no perioperatório e a longo prazo. Sua estimativa é passo importante para a tomada de decisões em pacientes com alto risco cardíaco.

A capacidade funcional é expressa em "equivalentes metabólicos" (METs), sendo classificada como excelente (>10 METs), boa (7-10 METs), moderada (4-6 METs) e pobre $(<4$ METs).

Figura 1. Avaliação cardíaca perioperatória para DAC. ${ }^{2}$

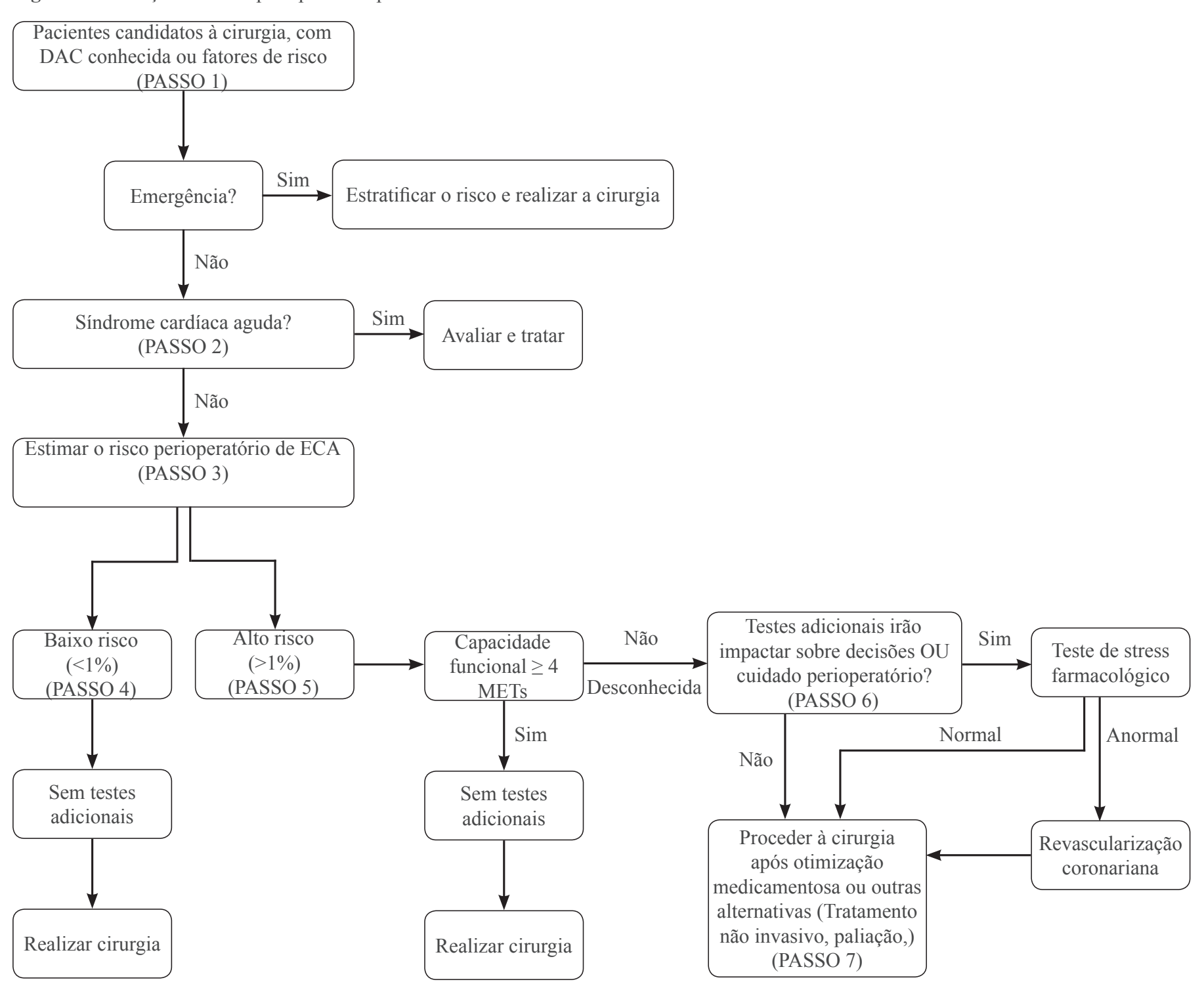

Fonte: elaborado pelos autores.

Diante de cirurgias de emergência, mesmo sendo o paciente portador de DAC ou de fatores de risco para tal, a avaliação pré-operatória deve-se limitar a testes e exames que não posterguem o procedimento (Classe I - Nível de evidência C).
Em estudo no qual avaliaram-se 600 pacientes submetidos a cirurgias não cardíacas, isquemia miocárdica perioperatória e outros ECAs foram mais comuns naqueles com pobre status funcional, mesmo após ajuste de outros fatores de risco. ${ }^{13}$

\section{Algoritmo de avaliação cardiovascular perioperatória}

Em 2014, o ACC e a AHA publicaram novas diretrizes de avaliação cardiovascular perioperatória de pacientes submetidos à cirurgia não cardíaca, com enfoque no screening de DAC. ${ }^{2} \mathrm{O}$ algoritmo proposto sistematiza e simplifica a avaliação, tornando-a mais aplicável à prática clínica diária (Figura 1). 


\section{Recomendações complementar \\ para \\ avaliação \\ pré-operatória}

Importante ressaltar que a solicitação de exames deve ponderar eficácia versus riscos. Estima-se que os custos individuais da avaliação cardiovascular pré-operatória reduziram-se drasticamente (U\$ 1.087 para U\$ 171) nos centros que adotaram as recomendações do ACC e da AHA. Paralelamente, os desfechos no pós operatório melhoraram, com queda na taxa de mortalidade por IAM de $11 \%$ para $4 \% .{ }^{15}$

\section{Eletrocardiograma de repouso de 12 derivações}

Não estão definidos idade padrão ou fator de risco decisivos que justifiquem a solicitação de rotina para o eletrocardiograma (ECG) de repouso no pré-operatório. Da mesma forma, não há consenso quanto ao intervalo entre a obtenção do ECG e a realização da cirurgia eletiva, parecendo ser adequado o intervalo de 1-3 meses em pacientes estáveis. ${ }^{2}$

- ECG é recomendado a pacientes com DAC, arritmia, doença arterial periférica, doença cerebrovascular e cardiopatia estrutural, exceto para procedimentos de baixo risco (Classe IIa - Nível de evidência: B);

- Pode ser considerado em indivíduos assintomáticos, sem DAC, exceto para aqueles submetidos a procedimentos de baixo risco (Classe IIb - Nível de evidência: B).

2. Avaliação da função ventricular esquerda

- Recomenda-se realização de ecocardiograma no pré-operatório de pacientes com dispneia de origem desconhecida e daqueles com IC definida, com piora da dispneia ou outra alteração do status clínico (Classe IIa - Nível de evidência: C);

- Pode-se considerar reavaliação com novo ecocardiograma naqueles pacientes com IC controlada se o exame não tiver sido realizado nos últimos 12 meses (Classe IIb - Nível de evidência: C).

3. Teste ergométrico para avaliação de isquemia miocárdica e capacidade funcional

- Para pacientes com elevado risco, porém com excelente capacidade funcional ( $>10$ METs), deve-se proceder à cirurgia sem teste ergométrico (Classe IIa - Nível de evidência: B);

- Naqueles sob elevado risco, com capacidade funcional desconhecida, pode-se considerar a realização de teste ergométrico caso os resultados possam mudar a conduta (Classe IIb - Nível de evidência: B);

- Pacientes com elevado risco e capacidade funcional pobre $(<4$ METs) ou desconhecida podem se beneficiar de testes de esforço com imagem cardíaca caso os resultados possam mudar a conduta (Classe
IIb - Nível de evidência: C);

- Testes ergométricos de rotina não são úteis a pacientes sob baixo risco no contexto de cirurgias não cardíacas (Classe III - Nível de evidência: B).

\section{Testes de stress farmacológico}

Testes de stress farmacológico têm um alto valor preditivo negativo para ECA, porém, baixo valor preditivo positivo: em média $12 \%$ para cintilografia miocárdica e $20 \%$ para o ecocardiograma de estresse com dobutamina. ${ }^{15}$ Assim, mesmo diante de teste positivo, a probabilidade de evento adverso cardiovascular no perioperatório é reduzida.

Metanálise publicada em 2006 identificou ligeira superioridade do ecocardiograma de stress com dobutamina em relação à cintilografia miocárdica na capacidade de predição de eventos cardíacos no pós operatório. ${ }^{16}$

- Teste de stress farmacológico (seja ecocardiograma de stress com dobutamina ou cintilografia miocárdica com dipiridamol/adenosina) é recomendado naqueles pacientes com pobre capacidade funcional e sob alto risco para cirurgias não cardíacas, caso haja algum impacto sobre a conduta final (Classe IIa - Nível de evidência: B);

- Testes de stress farmacológico de rotina não são úteis a pacientes sob baixo risco no contexto de cirurgias não cardíacas (Classe III - Nível de evidência: B).

Todos os testes de stress, ergométrico ou farmacológicos, devem ser evitados em pacientes instáveis.

\section{Angiografia coronariana}

- Em geral, as indicações para angiografia coronariana no pré-operatório são similares àquelas no cenário não cirúrgico, seguindo recomendações específicas. Portanto, não se recomenda angiografia coronariana pré-operatória de rotina (Classe III - Nível de evidência: C).

\section{Revascularização coronariana}

- Pacientes submetidos à estratificação de risco antes de procedimentos não cardíacos eletivos cuja avaliação recomenda cirurgia de bypass coronariano devem realizar revascularização antes da cirurgia previamente proposta (Classe I - Nível de evidência: C);

- Não se recomenda revascularização coronariana de rotina apenas como estratégia de redução de risco de eventos cardíacos no perioperatório (Classe III Nível de evidência: B).

Tempo de espera para cirurgias eletivas não cardíacas em pacientes com intervenção coronariana percutânea

- Cirurgias não cardíacas eletivas devem ser 
postergadas por 14 dias após angioplastia com balão (Classe I - Nível de evidência: C), por 30 dias após implante de stent metálico (SM) e por 365 dias após colocação de stent farmacológico (SF) (Classe I Nível de evidência: B);

- Em pacientes nos quais a cirurgia não cardíaca eletiva é necessária, devem-se ponderar os riscos clínicos e cirúrgicos relativos à suspensão ou manutenção da terapia antiplaquetária (Classe IIa - Nível de evidência: C);

- Cirurgia não cardíaca eletiva não deve ser realizada antes de 30 dias após implante de SM e dentro de 12 meses após SF em pacientes nos quais a dupla terapia de antiagregação plaquetária terá de ser interrompida no perioperatório (Classe III - Nível de evidência: B);

- Se os riscos de atraso na realização da cirurgia eletiva não cardíaca forem superiores aos riscos de isquemia e trombose do SF, a cirurgia pode ser considerada após 180 dias do implante do SF (Classe IIb - Nível de evidência: B);

- Se intervenção coronariana percutânea for necessária, urgência da cirurgia não cardíaca e risco de sangramento e eventos isquêmicos, incluindo trombose do stent, no paciente que faz uso de dupla terapia de antiagregação plaquetária, precisam ser considerados. Se há baixo risco de sangramento ou se a cirurgia pode ser postergada por 12 meses, colocação de SF é uma opção (optar por uso de aspirina e inibidores de receptor plaquetário $\mathrm{P} \mathrm{Y}_{12}$ );

- Se a cirurgia não cardíaca eletiva idealmente deve ocorrer entre 1 e 12 meses, pode ser uma estratégia adequada colocação de SM e 4-6 semanas de dupla antiagregação plaquetária, com manutenção de aspirina no perioperatório;

- Se a cirurgia não cardíaca for de urgência ou emergência, então os riscos de isquemia e sangramento, além dos benefícios a longo prazo da revascularização coronariana precisam ser considerados. Se a revascularização coronariana tiver indicação absoluta, bypass coronariano cirúrgico combinado à cirurgia não cardíaca podem ser consideradas.

\section{Terapia medicamentosa perioperatória}

\section{Beta bloqueadores}

O uso pré-operatório de $\beta$-bloqueadores foi associado à redução de eventos cardíacos em uma série de estudos analisados. Porém, poucos dados suportam a eficácia da administração pré-operatória de $\beta$-bloqueadores para reduzir os riscos de morte cirúrgica. Observaram-se, ainda, consistentes e claras associações entre administração de $\beta$-bloqueadores e desfechos adversos, como bradicardia, hipotensão e acidente vascular cerebral. ${ }^{17}$

- Beta bloqueadores devem ser mantidos naqueles pacientes cirúrgicos que fazem uso crônico prévio (Classe I - Nível de evidência: B);

- Para pacientes com estratificação de risco préoperatório intermediário a alto para isquemia miocárdica no teste de stress, parece ser válido introdução perioperatória de $\beta$-bloqueadores (Classe IIb - Nível de evidência: C). Nestes, a introdução deve ocorrer preferencialmente antes de 1 dia da cirurgia, a fim de avaliar a segurança e tolerabilidade (Classe IIb - Nível de evidência: B);

- Para pacientes com 3 ou mais fatores de risco pelo IRCR, pode ser útil iniciar $\beta$-bloqueadores antes da cirurgia (Classe IIb - Nível de evidência: B);

- Beta bloqueio não deve ser iniciado no dia da cirurgia (Classe III - Nível de evidência: B).

Lembrar que a suspensão abrupta do $\beta$-bloqueador naqueles pacientes que fazem uso crônico é deletéria.

\section{Estatinas}

- Deve-se manter estatinas naqueles pacientes que fazem uso crônico e estão aguardando cirurgias não cardíacas (Classe I - Nível de evidência: B);

- Naqueles pacientes que serão submetidos a cirurgias vasculares, recomenda-se iniciar estatinas no perioperatório (Classe IIa - Nível de evidência: B);

- Para pacientes que têm indicação clínica de uso de estatinas e que serão submetidos a procedimentos de alto risco, início no perioperatório pode ser considerado (Classe IIb - Nível de evidência: C).

\section{Alfa-2 agonistas}

Clonidina não reduz risco de IAM e de óbitos no perioperatório. Aumenta a incidência de parada cardíaca não fatal e hipotensão clinicamente importante. Porém, metanálise demonstrou redução significativamente estatística na mortalidade e na incidência de IAM dentre pacientes em uso de $\alpha_{2}$ agonistas submetidos a cirurgias vasculares. ${ }^{18}$

- Alfa-2 agonistas não são recomendados para prevenção de eventos cardíacos em pacientes candidatos à cirurgia não cardíaca (Classe III - Nível de evidência: B).

4. Bloqueadores de canais de cálcio

Permanece indefinido o real valor destes agentes no perioperatório. Sabe-se que aqueles com potenciais efeitos inotrópicos negativos, como verapamil e diltiazem, podem precipitar ou agravar IC em pacientes com baixa fração de ejeção e IC clínica. 
5. Inibidores da enzima de conversão de angiotensina

Em um grande estudo restrospectivo envolvendo 79.228 pacientes, maior incidência de hipotensão transitória foi observada nos usuários de inibidores da enzima de conversão de angiotensina (IECA), sem diferença quanto a outros desfechos. ${ }^{19}$

- É aceitável manter o uso de IECA e dos bloqueadores do receptor de angiotensina (BRA) no perioperatório (Classe IIa - Nível de evidência: B);

- Se IECAs ou BRAs são iniciados imediatamente antes da cirurgia, é prudente suspendê-los e reintroduzí-los tão logo possível no pós operatório (Classe IIa - Nível de evidência: C).

\section{Antiplaquetários}

$\mathrm{O}$ risco de trombose do stent no período perioperatório, tanto para SM quando para SF, é maior nas primeiras 4-6 semanas após o implante..$^{20}$

- Em pacientes submetidos à cirurgia não cardíaca de urgência durante as primeiras 4-6 semanas após implante de stent (metálico ou farmacológico), terapia de dupla antiagregação plaquetária deve ser mantida, exceto se o risco relativo de sangramento superar o benefício da prevenção de trombose do stent (Classe I - Nível de evidência: C);

- É recomendada manutenção da aspirina e suspensão do inibidor do receptor plaquetário $\mathrm{P}_{2} \mathrm{Y}_{12}$ (que deve ser reiniciado tão logo quanto possível no pós operatório) naqueles pacientes que serão submetidos à cirurgia que exija a suspensão deste (Classe I Nível de evidência: C);

- O manejo da terapia antiplaquetária deve ser determinado de forma consensual por cirurgião, anestesiologista, cardiologista e paciente, considerando o risco relativo de sangramento e de trombose do stent (Classe I - Nível de evidência: C);

- Para pacientes que fazem uso prévio de aspirina, parece ser razoável mantê-la quando o risco potencial de eventos cardíacos for superior ao risco de sangramento (Classe II - Nível de evidência: B) (Figura 2).

Figura 2. Algoritmo de manejo de terapia de antiagregação plaquetária em pacientes portadores de stent com proposta de cirurgia cardíaca. ${ }^{2}$

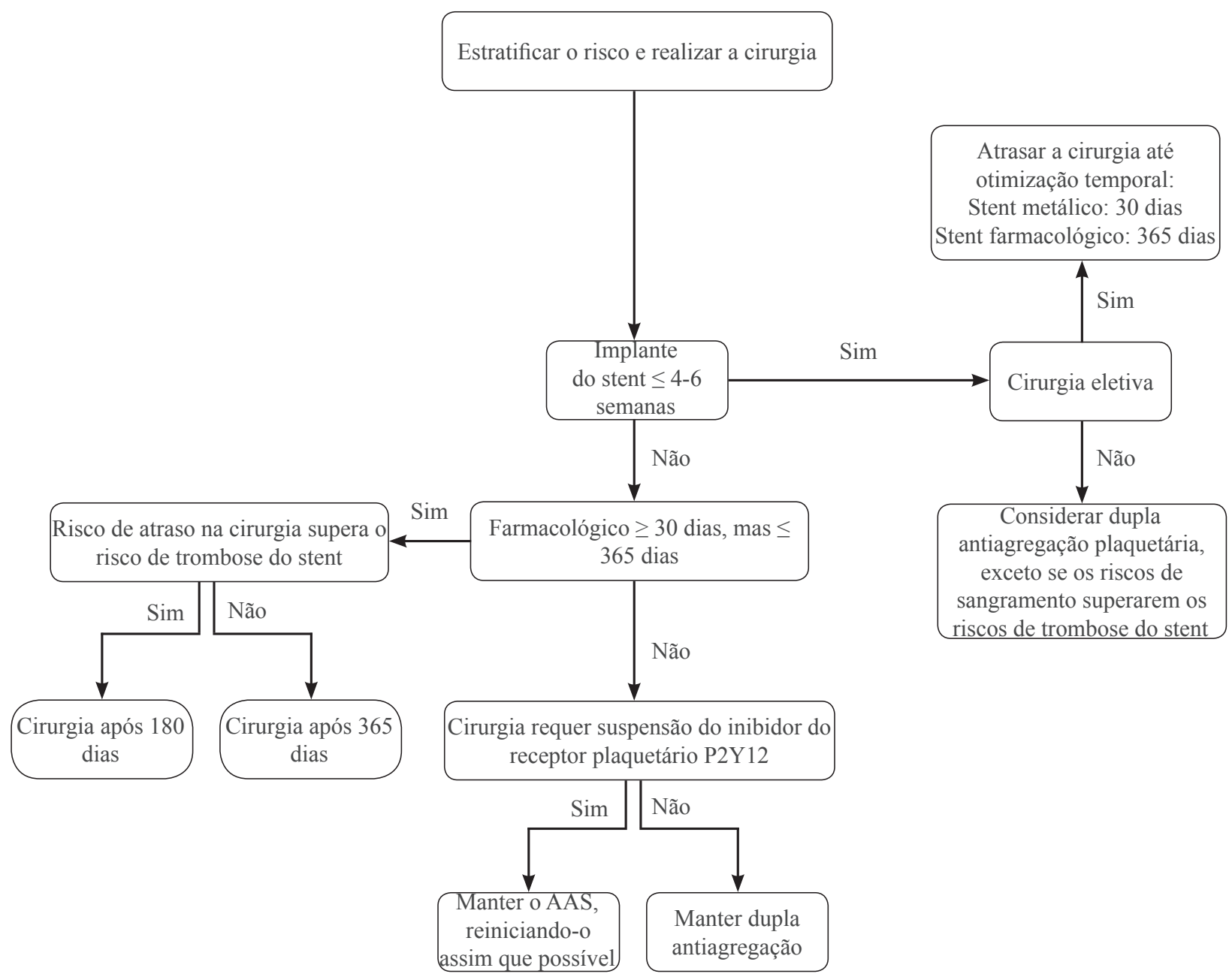

Fonte: elaborado pelos autores. 


\section{Anemia no perioperatório}

É bem estabelecido que anemia pode desencadear isquemia miocárdica, particularmente em pacientes coronariopatas. Em 2012, a American Association of Blood Banks publicou diretriz na qual recomenda estratégia de transfusão restritiva $(\mathrm{Hb}<7-8 \mathrm{~g} / \mathrm{dL})$ em pacientes assintomáticos, estáveis hemodinamicamente e sem DAC. Naqueles pacientes coronariopatas hospitalizados sugere transfusão apenas se sintomáticos ou com níveis de hemoglobina inferiores a $8 \mathrm{~g} /$ dL. No pós operatório, recomenda manter níveis de $\mathrm{Hb} \geq 8 \mathrm{~g} /$ $\mathrm{dL}$, exceto se paciente manifestar sintomas. ${ }^{21}$

\section{REFERÊNCIAS}

1. Buitrago FJ, Santana JA, Guimarães LF, Henriques MD, Almeida WM Jr. Avaliação cardiovascular perioperatória para cirurgia não cardíaca. Rev Med Minas Gerais. 2011;21(2 Supl 3):9-27.

2. Fleisher LA, Fleischmann KE, Auerbach AD, Barnason SA, Beckman JA, Bozkurt B, et al. 2014 ACC/AHA Guideline on perioperative cardiovascular evaluation and management of patients undergoing noncardiac surgery: executive summary. J Am Coll Cardiol. 2014;64(22):2373-2405.

3. Devereaux PJ, Goldman L, Yusuf S, Gilbert K, Leslie K, Guyatt GH. Surveillance and prevention of major perioperative ischemic cardiac events in patients undergoing noncardiac surgery: a review. CMAJ. 2005;173(7):779-88.

4. Badner NH, Knill RL, Brown JE, Novick TV, Gelb AW. Myocardial infarction after noncardiac surgery. Anesthesiology.1998;88(3):572-8.

5. Livhits M, Ko CY, Leonardi MJ, Zingmond DS, Gibbons MM, de Virgilio C. Risk of surgery following recent myocardial infarction. Ann Surgery. 2011;253(5):857-64.

6. Flu WJ, van Kuijk JP, Hoeks SE, Kuiper R, Schouten O, Goei $\mathrm{D}$, et al. Prognostic implications of asymptomatic left ventricular dysfunction in patients undergoing vascular surgery. Anesthesiology. 2010;112(6):1316-24.

7. Rohde LE, Polanczyk CA, Goldman L, Cook EF, Lee RT, Lee TH. Usefulness of transthoracic echocardiography as a tool for risk stratification of pacients undergoing major noncardiac surgery. Am J Cardiol.2001;87(5):505-9.

8. Matyal R, Hess PE, Subramaniam B, Mitchell J, Panzica PJ, Pomposelli F, et al. Perioperative diastolic dysfunction during vascular surgery and its association with postoperative outcome. J Vasc Surg. 2009;50(1):70-6.

9. Healy KO, Waksmonski CA, Altman RK, Stetson PD, Reyentovich A, Maurer MS. Perioperative outcome and long-term mortality for heart failure patients undergoing intermediate- and high-risk noncardiac surgery: impact of left ventricular ejection fraction. Congest Heart Fail. 2010;16(2):45-9.

10. Douglas PS, Garcia MJ, Haines DE, Lay WW, Manning WJ, Patel AR, et al. ACCF/ASE/AHA/ASNC/HFSA/HRS/SCAI/SCCM/ SCCT/SCMR 2011 appropriate use criteria for echocardiography: a report of the American College of Cardiology Foundation appropriate use criteria task force, American Society of Echocardiography, American Heart Association, American Society of Nuclear

\section{CONCLUSÃO}

Anualmente, inúmeras publicações surgem com o objetivo de sistematizar e tornar aplicáveis instrumentos de avaliação cardiovascular no perioperatório de cirurgias não cardíacas. Dessa forma, há uma tendência à redução na incidência de morbimortalidade associada a eventos cardíacos adversos, redução de custos com hospitalização e solicitação abusiva de exames complementares, além de menores taxas de suspensão de procedimentos eletivos.

Cardiology, Heart Failure Society of America, Heart Rhythm Society, Society for Cardiovascular Angiography and Interventions, Society of Critical Care Medicine, Society of Cardiovascular Computed Tomography, and Society for Cardiovascular Magnetic Resonance endorsed by the American College of Chest Physicians. J Am Coll Cardiol. 2011;57(9):1126-66.

11. Agarwal S, Rajamanickam A, Bajaj NS, Griffin BP, Catacutan $\mathrm{T}$, Svensson LG, et al. Impact of aortic stenosis on postoperative outcomes after noncardiac surgeries. Circ Cardiovasc Qual Outcomes. 2013;6(2):193-200.

12. Lee TH, Marcantonio ER, Mangione CM, Thomas EJ, Polanczik $\mathrm{CA}$, Cook EF, et al. Derivation and prospective validation of a simple index for prediction of cardiac risk of major noncardiac surgery. Circulation. 1999;100(10):1043-9.

13. Reilly DF, McNeely MJ, Doerner D, Greenberg DL, Staiger TO, Geist MJ, et al. Self-reported exercise tolerance and the risk of serious perioperative complications. Arch Intern Med. 1999;159(18):218592.

14. Fleisher LA, Beckman JA, Brown KA, Calkins H, Chaikof EL, Fleischmann KE, et al. 2009 ACCF/AHA focused update on perioperative beta blockade incorporated into the ACC/AHA 2007 guidelines on perioperative cardiovascular evaluation and care for noncardiac surgery. J Am Coll Cardiol. 2009;54(22):e13-e118.

15. Froehlich JB, Fleisher LA. Noncardiac surgery in the patient with heart disease. Anesthesiol Clin. 2009;27(4):649-71.

16. Beattie WS, Abdelnaem E, Wijeysundera DN, Bucley DN. A meta-analytic comparison of preoperative stress echocardiography and nuclear scintigraphy imaging. Anesth Analg. 2006;102(1):8-16.

17. Wijeysundera DN, Duncan D, NKonde-Price C, Virani SS, Washam JB, Fleischmann KE, et al. Perioperative beta blockade in noncardiac surgery: a systematic review for the 2014 ACC/AHA guideline on perioperative cardiovascular evaluation and management of patients undergoing noncardiac surgery: a report of the American College of Cardiology/American Heart Association Task Force on practice guidelines. J Am Coll Cardiol. 2014;64(22):2406-25.

18. Wijeysundera DN, Naik JS, Beattie WS. Alpha-2 adrenergic agonists to prevent perioperative cardiovascular complications: a meta-analysis. Am J Med. 2003;114:742-52.

19. Turan A, You J, Shiba A, Kurz A, Saager L, Sessler DI. Angiotensin converting enzyme inhibitors are not associated with 
respiratory complications or mortality after noncardiac surgery. Anesth Analg. 2012;114(3):552-60.

20. Kaluza GL, Joseph J, Lee JR, Raizner ME, Raizner AE. Catastrophic outcomes of noncardiac surgery soon after coronary stenting. J Am Coll Cardiol. 2000;35(5):1288-94.
21. Carson JL, Grossman BJ, Kleinman S, Tinmouth AT, Marques MB, Fung MK, et al. Red blood cell transfusion: a clinical practice guideline from the AABB. Ann Intern Med. 2012;157(1):49-58.

\section{Como citar:}

Silva DR, Cavalcante FP, Maia FL, Oliveira LA, Fernandes CR. Avaliação cardiovascular perioperatória em cirurgias não cardíacas como ferramenta para redução de risco de eventos cardíacos adversos. Rev Med UFC. 2016 jul-dez;56(2):30-38. 